\title{
Féeries
}

Études sur le conte merveilleux, XVII $-\mathrm{XIX}{ }^{\mathrm{e}}$ siècle

\section{Le jeu de l'anecdote et du conte dans les Mémoires du duc de Saint-Simon}

The Game of Anecdote and Tale in Saint-Simon's Memoirs

\section{Marc Hersant}

\section{(2) OpenEdition}

1 Journals

\section{Édition électronique}

URL : http://journals.openedition.org/feeries/699

DOI : $10.4000 /$ feeries.699

ISSN : 1957-7753

\section{Éditeur}

UGA Éditions/Université Grenoble Alpes

\section{Édition imprimée}

Date de publication : 1 juillet 2009

Pagination : 47-62

ISBN : 978-2-84310-140-3

ISSN : 1766-2842

\section{Référence électronique}

Marc Hersant, « Le jeu de l'anecdote et du conte dans les Mémoires du duc de Saint-Simon », Féeries [En ligne], 6| 2009, mis en ligne le 15 septembre 2010, consulté le 08 septembre 2020. URL : http:// journals.openedition.org/feeries/699; DOI : https://doi.org/10.4000/feeries.699

\section{(c) Féeries}




\section{LE JEU DE L'ANECDOTE ET DU CONTE DANS LES MÉMOIRES DU DUC DE SAINT-SIMON}

$\Gamma$

es MémoIREs de Saint-Simon ', d'un style narratif si remarquablement hétéroclite qu'il défie l'idée même de "style individuel ", semblent absorber et faire dialoguer, dans l'unité de principe d'une chronique, presque toutes les formes de récits ou de "relations " qu'on peut trouver ailleurs dans les textes de l'époque classique. Ainsi, les comptes-rendus des cérémonies princières y ont-ils souvent l'allure sèchement informative des gazettes ${ }^{2}$ ou du Journal de Dangeau, officiellement méprisés par l'auteur, alors que les intrigues de la cour de Savoie autour de la comtesse de Verue ${ }^{3}$ ou celles du mariage du prince de Léon ${ }^{4}$ semblent tout droit sorties des nouvelles historiques à la mode du siècle précédent. La hargne austère des enquêtes généalogiques', imitée de spécialistes célèbres comme Imhof, côtoie la sérénité " touristique » des récits de voyage en Espagne ${ }^{6}$. Les récits militaires, généralement voués à dénoncer les "bévues" des bêtes noires de Saint-Simon", sont écrits dans un style faussement objectif parfois rebutant, fonctionnant en réalité comme réquisitoire. Celui-ci tranche singulièrement avec les passages les plus souvent assimilés par la critique à une écriture "romanesque ", comme si ce constat valait pour la totalité de l'œuvre, tel le fameux récit, dans la chronique de l'année $17 \mathrm{I} 5$, de la disgrâce de la princesse des Ursins ${ }^{8}$. L'œuvre

I. Toutes les références aux Mémoires se feront ici dans l'édition d'Yves Coirault, Gallimard, "Bibliothèque de la Pléiade ", 8 volumes, 1983-1988.

2. Voir, parmi mille autres exemples, les cérémonies de réception de l'ambassadeur extraordinaire du Grand Seigneur à la cour, en VII, p. 774-775.

3. I, p. 747-750.

4. III, p. I54-I63.

5. On en découvrira un exemple harassant, à propos des Rohan, en I, p. 498-522.

6. Voir, par exemple, en VIII, p. 426.

7. Exemple archétypal : la bataille de Friedlingen, en II, p. 247-252.

8. V, p. I6I, pour le passage le plus caractéristique. 
d'histoire " particulière" " apparaît donc ici comme un " collage " qui n'a pas besoin de cohérence formelle. Loin de fonctionner comme un récit unitaire, elle se présente comme une juxtaposition "sérielle " presque infinie et toujours évidemment ouverte de récits divers ${ }^{10}:$ par leur format (de quelques lignes aux dimensions d'un bref roman); par le degré d'accomplissement de la " mise en intrigue " dans leur configuration; par leur registre (du sérieux le plus ténébreux au comique le moins raffiné) ; par leur rapport (plus ou moins serein ou tendu) avec la vérité ou avec le lecteur "représenté ", lui-même extraordinairement "transformiste "; par la place importante, réduite ou inexistante de "procédés " (comme la " transparence intérieure ") considérés par certains théoriciens ${ }^{\text {II }}$ comme caractéristiques de la fiction, etc. La prose chatoyante des Mémoires semble vouloir faire la synthèse de toutes les " paroles narratives " à l'œuvre dans le monde qu'ils représentent. Et dans cette œuvre globalement sombre et solitaire, les « éclats » d'une parole « sociale » détendue, souvenir des échanges spirituels de Saint-Simon avec d'autres courtisans, expliquent la place importante de la sphère narrative de l'anecdote, certaines parties de l'œuvre semblant poursuivre sans interlocuteur immédiat le « bavardage » archétypal d'un courtisan racontant pour briller, éblouir, étonner ${ }^{12} . \mathrm{La}$ nostalgie de la parole "mondaine " occupe donc sa place, et une place essentielle, dans le kaléidoscope énonciatif et formel des Mémoires, à côté de modèles «journalistiques " ou " érudits ", tant cette écriture solitaire ${ }^{13}$

9. C'est ainsi, on le rappelle, que Saint-Simon, utilisant les catégories de son temps, caractérise son œuvre dans la "préface " de ses Mémoires, par opposition à l'histoire " générale " (I, p. 6-7).

Io. On trouvera un examen plus approfondi de cette hétérogénéité de l'œuvre dans la partie intitulée "Les styles de la vérité " de ma thèse, Le discours de vérité dans les Mémoires du duc de Saint-Simon, Honoré Champion, 2008.

II. Käte Hamburger et, après elle, Dorrit Cohn.

I2. La place de l'anecdote dans la conversation à l'époque classique et plus particulièrement dans la parole de cour est désormais bien connue, et l'anecdote apparait clairement comme un des lieux de rencontre entre la culture de la conversation et l'écriture de l'histoire, surtout quand cette dernière est écrite par des hommes du "monde ". Les liens entre anecdote et mot d'esprit dans la culture de cour d'Ancien Régime mériteraient en revanche un approfondissement tant l'anecdote apparaît souvent comme une expansion narrative du bon mot, tant le " mot " contient le plus souvent un potentiel narratif (celui d'une narration à " reconstituer " par l'auditeur, par exemple). L'anecdote participe en outre à une écriture de l'histoire « myope » rivée à ce que Voltaire appellera avec dédain des " détails " dignes de la conversation d'une " femme de chambre ", particulièrement dans le "potinier " des Mémoires d'Ancien Régime. L'anecdote est donc chez Saint-Simon au carrefour d'une culture de cour, d'une culture de la « conversation » et d'une conception " aristocratique » de l'histoire, et ces traits traversent tout l'Ancien Régime.

I3. Sur la "solitude " de Saint-Simon, et sans pour autant cautionner une lecture " romantique " de son œuvre, on consultera la belle présentation d'Yves Coirault, "Solitude de Saint-Simon ", Manuel d'histoire littéraire de la France, Paris, Éditions Sociales, I969, t. III, p. 385-392, et surtout 
porte avec elle le «fantôme " de toutes les paroles sociales. Or, à l'époque de la jeunesse et de la maturité de Saint-Simon ${ }^{14}$, la mode du "conte merveilleux » avait atteint les plus hautes sphères de la cour et du pouvoir et fonctionné comme un des éléments les plus en vogue du "badinage " des courtisans, ce dont témoignent un épisode célèbre des Mémoires attribuant à Madame un conte de fées censé expliquer le caractère du duc d'Orléans ${ }^{15}$ et un dialogue politique entre Saint-Simon et le duc de Noailles où le premier taxe les projets du second d'extravagances qui nécessiteraient l'intervention des fées ${ }^{16}$. Il n'est donc pas étonnant que le conte joue un rôle relativement discret mais significatif dans le " dialogue des récits " qui donne à l'œuvre historique du mémorialiste son allure de mosaïque, et que le genre narratif oral le plus pratiqué par les courtisans, l'anecdote, rencontre, sur un mode plus ou moins plaisant, le conte, et singulièrement le conte de fées. Car Saint-Simon, qui possédait dans sa bibliothèque les œuvres de $\mathrm{M}^{\mathrm{me}} \mathrm{d}$ 'Aulnoy aussi bien que les Mille et Une Nuits, malgré son aversion officielle pour les " romans ", n'y avait pas été insensible. Cela ne signifie pas qu'on trouve dans les Mémoires des " contes merveilleux » à proprement parler. Mais le modèle narratif le plus directement lié à la parole de cour parmi tous ceux qui se retrouvent dans les Mémoires, celui de l'anecdote, "flirte" souvent, plus ou moins consciemment et plus ou moins ironiquement, avec l'univers narratif du conte, en retrouvant certains de ses motifs, de ses lieux archétypaux, de ses personnages, ainsi que la " naïveté » réelle ou feinte de son écriture. Et si les études ont proliféré depuis quelques années sur l'anecdote aussi bien

l'essai d'Alphonse de Waelhens, Le duc de Saint-Simon "immuable comme Dieu et d'une suite enragée ", Bruxelles, Publications des Facultés Universitaires Saint-Louis, 198I.

I4. Né en 1775 .

I5. En V, p. 245 : «Madame était pleine de contes et de petits romans de fées. Elle disait qu'elles avaient toutes été conviées à ses couches, que toutes y étaient venues, et que chacune avait doué son fils d'un talent, de sorte qu'il les avait tous; mais que par malheur on avait oublié une vieille fée disparue depuis si longtemps qu'on ne se souvenait plus d'elle, qui, piquée de l'oubli, vint appuyée sur son petit bâton et n'arriva qu'après que toutes les fées eurent fait chacune leur don à l'enfant ; que, dépitée de plus en plus, elle se vengea en le douant de rendre absolument inutiles tous les talents qu'il avait reçus de toutes les autres fées, d'aucun desquels, en les conservant tous, il n'avait jamais pu se servir. " Notons que ce " conte ", confirmé par la princesse Palatine qui l'affectionne au point de le reprendre dans plusieurs de ses lettres, joue au moins un des rôles les plus fréquents de l'anecdote : "caractériser " un personnage. Il fonctionne comme un " conte des origines " du caractère du duc d'Orléans, et en même temps comme le substitut d'une anecdote (par exemple, sur l'enfance du prince) qui prétendrait donner à voir l'essence de son caractère.

I6. En V, p. 405. Saint-Simon écoute les élucubrations de son interlocuteur qui veut (entre autres) détruire Versailles, puis reprend : «Monsieur [...] pour ce que vous me proposez, il nous faut les fées. Jusqu’à ce que vous les ayez en main, il n'y a pas moyen d'en raisonner. " 
que sur le conte, les liens entre ces deux "formes simples » de narrations brèves, particulièrement intéressants pour qui s'intéresse aux rapports entre narration historique (au sens large) et narration fictionnelle à l'époque classique, mais aussi au lien entre les formes « littéraires » et les formes " mondaines » de la parole, reste, pour l'essentiel ${ }^{17}$, à interroger.

Il n'est d'ailleurs pas certain qu'anecdote et conte soient aussi nettement distincts dans les représentations de Saint-Simon et de ses contemporains que dans nos classifications génériques pour une large part fabriquées a posteriori, comme peut le montrer une brève réflexion sur l'usage du mot " conte " dans ses Mémoires, qui semble se distribuer en trois sens principaux au "continuum " manifeste ${ }^{18}$. Au sens de "conte de fées", ou même au sens plus large d'un genre " littéraire " narratif " comique », tel que l'entendra Marmontel ${ }^{19}$, l'emploi est on ne peut plus rare : on note une occurrence à propos de Madame, " pleine de contes et de petits romans de fée ${ }^{20}$ ", dans le passage auquel j'ai déjà fait allusion, et la mention de "contes", à côté des fables parmi les œuvres de La Fontaine, dans les maigres lignes qui lui sont consacrées à l'occasion de sa mort ${ }^{21}$. Les occurrences sont sans surprise beaucoup plus nombreuses d'un emploi de "conte " au sens de pur mensonge "narratif " : c'est le cas, entre beaucoup d'autres exemples, dans un passage qui dénonce les calomnies d'Alberoni contre le duc de Bourgogne en pleine campagne de Flandres ${ }^{22}$, dans un autre qui défend Rancé contre des versions dégradantes de l'origine de sa retraite pieuse ${ }^{23}$, dans un troisième où sont présentées comme des « contes » les histoires faites au Roi de conversions enthousiastes de

17. On mentionnera toutefois un article de Gianni Ioti qui interroge, en un sens un peu différent, les rapports entre écriture du conte et écriture de l'histoire chez Voltaire : "Le Siècle de Louis XIV : de l'histoire et de la fiction ", Voltaire et le Grand Siècle, Voltaire Foundation, Oxford, 2006, p. 8I-96.

18. Chez Furetière et dans le dictionnaire de l'Académie de 1740, les occurrences saint-simoniennes sont " confirmées" par les usages du temps : le point commun de tous les sens répertoriés de "conte ", c'est qu'il s'agit d'un récit sans valeur de vérité ou à valeur de vérité "atténuée " ou "dédramatisée ".

19. P. 302, dans les Éléments de littérature, éd. Sophie Le Ménahèse, Desjonquères, 2005 : «Le conte est à la comédie ce que l'épopée est à la tragédie, mais en petit [...]. »

20. V, p. 245 .

2I. I, p. 229 : «si connu par ses fables et ses contes, et toutefois si pesant en conversation ».

22. III, p. 209 : «De ce joli petit conte, et si bien inventé [...]. » Dans le même passage les mots " conte ", « fable » et « roman » se succèdent pour dénoncer les mensonges d'Alberoni dans sa lettrepamphlet.

23. I, p. 52I : « cette belle Madame de Montbazon dont on a fait ce conte, qui a trouvé croyance, que l'abbé de Rancé [...]. » C'est la célèbre anecdote de la découverte de la tête coupée de sa maîtresse par le futur abbé de La Trappe. 
Protestants pendant la révocation de l'Édit de Nantes. Mais l'emploi le plus fréquent, et de très loin, renvoie à une parole narrative orale de cour avec quelques traits récurrents qui sont précisément ceux de l'anecdote : récit supposé " caractériser » un personnage, récit possédant un caractère " plaisant » et recherchant un " effet " comparable à celui d'un mot d'esprit, récit manifestant un art oral de la narration apprécié en "société ". Le catalogue des exemples possibles voisine ici avec l'infini. Louis XIV est ainsi loué par Saint-Simon, qui lui attribue pourtant ailleurs, on le sait, un esprit "au-dessous du médiocre ${ }^{24}$ ", de faire " un conte mieux qu'homme du monde, et aussi bien un récit ${ }^{25}$ ", et Philippe V, d'une intelligence encore beaucoup moins vantée par le mémorialiste, semble pourtant avoir hérité de ce talent de narrateur ${ }^{26}$. Louis XIV et Villeroi communient dans les "cent contes de leur jeunesse et de leur temps ${ }^{27}$ ". Boudin fait de lui-même "les contes les plus comiques ${ }^{28}$ ". Vivonne amuse le Roi de «cent contes meilleurs les uns que les autres qu'il se plaisait à raconter ${ }^{29}$ ". Et souvent Saint-Simon possède un tel réservoir de "contes » sur tel de ses modèles qu'il renonce à les rapporter tous : il court ainsi sur $\mathrm{M}^{\mathrm{me}}$ de Navailles « mille contes plus étranges et plus plaisants les uns que les autres de son avarice, trop nombreux à rapporter ${ }^{30} »$. Ces différents sens ne sont évidemment pas sans rapport, l'ensemble des occurrences recouvrant des modalités de récit entretenant un rapport suspendu (le " conte" au sens " littéraire " ou " fictionnel »), détendu (le " conte " au sens d'" anecdote ") ou vicié (le " conte " au sens de " mensonge ") à la vérité. En particulier, la frontière entre le «conte » comme fiction et le " conte " comme anecdote paraît on ne peut plus floue, précisément parce que la sphère de l'anecdotique est celle, non d'une suppression, mais d'une « détente » du rapport avec la question de la vérité de la parole narrative, qui semble pouvoir produire des formes proches de celles qui sont engendrées par une suspension pure et simple de ce rapport, et parce que le récit anecdotique, autant que le conte « fictionnel », cherche à plaire, et

24. V, p. 469.

25. V, p. 480.

26. VIII, p. 268 : «D'ailleurs c'était l'homme du monde qui remarquait mieux les défauts et les ridicules, et qui en faisait un conte le mieux dit et le plus plaisant. »

27. IV, p. 446.

28. III, p. I042.

29. III, p. 366. Le lien avec la culture du mot d'esprit est, dans le contexte, particulièrement patent, Vivonne en étant une vraie fontaine : "C'était l'homme le plus naturellement plaisant, et avec le plus d'esprit et de sel et le plus continuellement, dont j'ai ouï faire au feu roi cent contes meilleurs les uns que les autres qu'il se plaisait à raconter. "

30. I, p. 694. On pourrait sans peine multiplier les exemples...

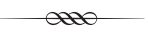


utilise les artifices qui assurent la séduction d'un récit. C'est d'ailleurs une des raisons pour lesquelles l'anecdote a eu beaucoup de mal à s'imposer en historiographie : si elle ne parait pas "sérieuse ", ce n'est pas seulement parce qu'elle s'attarde sur des «bagatelles " qu'un Voltaire ${ }^{3 \mathrm{I}}$ jugera indignes de l'étude de "l'esprit humain ", mais aussi parce qu'elle s'apparente, d'un point de vue formel aussi bien que dans son régime énonciatif, à ce que nous appelons aujourd'hui, dans un sens restreint, " conte ». Une des conséquences de ce voisinage, qu'on ne développera pas ici, est que les nombreux clins d'œil aux personnages archétypaux du conte de fées qu'on trouve dans les Mémoires ${ }^{32}$, observés par Yves Coirault dans son Optique de Saint-Simon ${ }^{33}$, ne se rencontrent que dans les parties de l'œuvre où la verve " anecdotique " se déploie : c'est dans ces parages qu'on trouvera les multiples assimilations des personnages réels des Mémoires à des "fées ", à des "sorcières " ou, plus exceptionnellement, à des " ogres ${ }^{34}$ ». Ces jeux seraient totalement incongrus dans les récits militaires, dans les enquêtes généalogiques ou dans les passages de style " gazette ", et la proximité de l'anecdote et du conte (aux sens actuels) s'observe déjà dans cette facilité de l'anecdote à assimiler, même si c'est ironiquement, des éléments venus du conte. Cela tient à la "détente » commune aux deux genres : si le conte est pure "fiction " éliminant d'emblée toute valeur de vérité, l'anecdote est le type de récit "factuel " le plus " détendu » dans son rapport avec la vérité, celui qui se rapproche le plus du simple plaisir de raconter une " histoire » et où la crispation sur la valeur de vérité de cette histoire est la moins grande. L'esprit de «badinage » de l'anecdote est donc susceptible de tolérer des éclats métaphoriques plaisants empruntés à ce «badinage pur » qu'est, aux yeux du courtisan, le conte merveilleux.

Dans certains cas, l'assimilation d'un des acteurs " réels " des Mémoires à un personnage de conte de fées semble déteindre sur l'ensemble des récits qui lui sont associés, la psychologie du personnage se réduisant en peau de chagrin à une " épure " et le style narratif devenant particuliè-

3I. Qui n'en consacre pas moins des chapitres entiers aux anecdotes dans son Siècle de Louis XIV.

32. Nettement plus fréquents, par exemple, que les assimilations à des personnages « mythologiques".

33. Arman Colin, 1965. Voir aussi les analyses de François Raviez dans Le Duc de Saint-Simon et l'Écriture du mal. Une lecture démonologique des Mémoires, Honoré Champion, 2000 et mon propre article "L'historien et le conteur : Histoire et merveilleux dans les récits de l'époque classique (Perrault, Saint-Simon) ", à paraître dans XVII siècle.

34. Voir, sur ce point, les relevés précis des occurrences dans mon article "L'historien et le conteur ", à paraître dans XVII siècle. 
rement « minimal » et " pseudo-populaire ». Ainsi, $\mathrm{M}^{\text {me }}$ d'Heudicourt comparée à une "mauvaise fée qui ne savait que nuire ${ }^{35}$ " devient-elle le foyer d'une succession d'anecdotes où toute l'extraordinaire complexité dont est capable ailleurs Saint-Simon a totalement disparu ; "vieille et hideuse " comme une sorcière, sa psychologie est des plus rudimentaires et étroitement associée à son physique : "On ne pouvait aussi être plus gratuitement, plus continuellement, plus désespérément méchante [...].» Son mari, qui ne connaît pas un traitement plus raffiné, " en tirait parti le bâton haut, sans presque vivre avec elle; mais il s'en était fait craindre. C'était un vieux vilain, fort débauché et horrible [...] ». Quant au fils, " c'était une manière de chèvre-pied, aussi méchant et plus laid encore que son père ${ }^{36}$ ". L'univers dans lequel cette petite troupe de personnages est transportée semble à mi-chemin entre le conte de fées et le fabliau, et, comme on peut l'observer, le style suit. Cette simplicité " excessive ", accompagnée d'un pastiche de parler populaire, fonctionne comme un signal de relâchement de la "tension » de vérité qui anime l'œuvre. Nous ne sommes pas dans un "conte de fées " à proprement parler, ni dans un fabliau, pas plus que nous ne sommes dans un roman dans les parties de l'œuvre les plus proches d'un style romanesque, mais l'univers représenté et les formes de la parole narrative " anecdotique " se sont considérablement rapprochés de ces modèles de référence. On fera le même constat pour la merveilleuse petite série d'anecdotes grotesques gravitant autour de $\mathrm{M}^{\mathrm{me}}$ de Saint-Herem, dont voici le début :

Cette $\mathrm{M}^{\mathrm{me}}$ de Saint-Herem était la créature du monde la plus étrange dans sa figure, et la plus singulière dans ses façons. Elle se grilla une fois une cuisse au milieu de la rivière de Seine, auprès de Fontainebleau, où elle se baignait : elle trouva l'eau trop froide ; elle la voulut chauffer, et pour cela elle en fit bouillir quantité au bord de l'eau, qu'elle fit verser tout auprès d'elle et au-dessus, tellement qu'elle en fut brûlée, à en garder le lit, avant que cette eau pût être refroidie dans celle de la rivière. Quand il tonnait elle se fourrait à quatre pattes sous un lit de repos, puis faisait coucher tous ses gens dessus l'un sur l'autre en pile, afin que, si le tonnerre tombait, il eût fait son effet sur eux avant de pénétrer jusqu'à elle. Elle s'était ruinée, elle et son mari qui étaient riches, par l'imbécillité, et il n'est pas croyable ce qu'elle dépensait à se faire dire des évangiles sur la tête ${ }^{37}$.

Où sont passés les abîmes "dostoïevskiens " des pages sur le duc d'Orléans ou le duc de Noailles et la formidable capacité de Saint-Simon

35. III, p. 347 .

36. III, p. 348 .

37. II, p. 43 . 
à capter le réel dans le foisonnement des singularités qu'il produit ? Et les autres "sorcières ", "fées ", "harpies ${ }^{38}$ " de Saint-Simon, comme $\mathrm{M}^{\mathrm{me}}$ de Montchevreuil, $\mathrm{M}^{\mathrm{me}}$ de Lesdiguières, $\mathrm{M}^{\mathrm{lle}}$ Balbien, $\mathrm{M}^{\mathrm{me}}$ Panache, la princesse d'Harcourt, $\mathrm{M}^{\mathrm{me}}$ de Miossens et tant d'autres, sont associées à des récits présentant les mêmes caractéristiques, constituant un des " univers narratifs" les plus facilement identifiables des Mémoires, qui peut même se greffer à l'occasion sur des personnages aussi complexes par ailleurs que $\mathrm{M}^{\mathrm{me}}$ de Maintenon ou $\mathrm{M}^{\text {lle }}$ Choin, dans les parages de leur assimilation à des figures de contes ${ }^{39}$. Ces " créatures " ne semblent pas pouvoir mériter une parole plus raffinée et paraissent au contraire susciter tout naturellement ce qui dans les Mémoires se rapproche le plus d'un pastiche - volontaire ou non - de la langue " populaire » et des " genres » auxquels elle est plus ou moins fantasmatiquement associée, comme si Saint-Simon devenait ici ou là une espèce de double masculin de la Mèregrand de la scène conteuse archétypale.

Si l'anecdote ne devient pas à la lettre un conte de fées, elle en prend donc les couleurs, et cette relation est susceptible d'être observée chez Saint-Simon dans les deux sens : on trouve dans les Légères notions des commandeurs, chevaliers et grands officiers de l'ordre du Saint-Esprit, œuvre écrite comme tant d'autres par le Saint-Simon hyper productif des années I730, un " récit " fabuleux au contenu narratif très proche des contes féeriques du temps ${ }^{40}$, que j'ai déjà eu l'occasion de commenter pour d'autres raisons ${ }^{41}$, et à laquelle Saint-Simon semble croire à moitié, hésitant tout au moins sur sa valeur de vérité. Le personnage principal, proche parent du comte de Cheverny, y rencontre dans une forêt un " génie ", espèce de gnome ou de sylphe, qui aurait pu lui porter bonheur, mais que sa crainte

38. Ce n'est pas un personnage de conte de fées, mais Saint-Simon semble à peine faire la différence.

39. Par exemple, en V, p. 368, et à propos de $\mathrm{M}^{\mathrm{me}}$ de Maintenon, dans un passage, comme tant d'autres, haineux : "Il n'y avait plus rien à craindre de cette fée presque octogénaire : sa puissante et pernicieuse baguette était brisée, elle était redevenue la vieille Scarron. "

40. Traités politiques et autres écrits, éd. Yves Coirault, Gallimard, "Bibliothèque de la Pléiade ", 1996, p. 892-893. Je ne citerai ici, pour mémoire, que la partie centrale de ce "conte " : "Il ne fut pas longtemps dans l'allée et au lieu indiqué, où il se promenait en attendant, qu'il vit à cent ou cent vingt pas une petite figure d'environ demi-pied de haut. Dès qu'il l'aperçut, il la vit, sans courir ni s'élever de terre, venir à lui rasant la terre avec une extrême agilité, et entendit un son de voix très claire et enfantine, mais forte. À cet aspect, la peur lui prit et le fit reculer, et, dans l'instant, cette petite figure retourna quelques pas et disparut. Demeuré seul, il rappela ses esprits, se repentit de sa frayeur, et en craignit les suites. Il s'avança vers le lieu où il avait vu le génie; mais il eut beau faire, il ne revint plus. "

4I. Dans mon article déjà cité, «L'historien et le conteur : Histoire et merveilleux dans les récits de l'époque classique (Perrault, Saint-Simon) ", à paraître dans XVII siècle. 
transforme au contraire en porte-malheur définitif. Or, alors que les styles de Saint-Simon, comme on l'a vu, sont extraordinairement divers, rien ne distingue le style particulier (format narratif, registres employés, univers de référence...) de ce récit de celui de mille " anecdotes " qu'on trouvera dans ces Légères notions comme dans les Mémoires et ailleurs dans l'immense production du mémorialiste : l'ambiguïté fascinante de ce texte tient peut-être, précisément pour des raisons de croyance, au fait que ce qui nous parait relever du conte de fées est en réalité, pour Saint-Simon, ce que nous appelons une anecdote, ou pourrait en être une. Les faits y sont relatés exactement comme s'ils étaient réels et la justification même du récit (l'auteur évoquant la " singularité ${ }^{42}$ " de ce qu'il a à rapporter) est la même que celle d'anecdotes extravagantes mais ne comportant aucun élément surnaturel des Additions à Dangeau et des Mémoires. Il faut dire que dans les Mémoires en particulier, Saint-Simon, partiellement touché par l'esprit des premières Lumières et par une pensée "semi critique ", semble s'être presque complètement interdit ce type de " dérapages ${ }^{43}$ ".

Ce qu'on trouve en revanche dans le kaléidoscope des " mille sketches divers " du vivier anecdotique des Mémoires, c'est un envahissement occasionnel de l'anecdote par le monde du conte, par son imaginaire et par sa langue, comme si l'univers du conte " remontait » ici ou là, dans cette vaste et extravagante œuvre d'histoire, par un clin d'œil ironique ou un détail rêveur. Ce sont parfois les " prodiges " de la vie de cour et du luxe des courtisans qui suscitent des comparaisons explicites avec la magie des contes. La magnificence inouïe du camp de Compiègne, conçu pour impressionner l'Europe et faire éclater la puissance de Louis XIV, suscite la dérision de Saint-Simon, mais aussi des images qui semblent directement provenir d'un conte, notamment dans l'évocation du luxe inouï déployé par le maréchal de Boufflers, dans une vision de pays de Cocagne ${ }^{44}$. L'effet est plus frappant encore, et surtout plus explicite, dans le récit de la visite du roi à Petit-Bourg, où d'Antin réalise ce que Saint-Simon nomme précisément dans la manchette des "prodiges de courtisan ", qui appellent bientôt la comparaison avec la magie des contes. L'auteur évoque le luxe et la profusion inouïe de cet accueil exceptionnel, avant de raconter un trait particulièrement extravagant qui suscite un rapprochement explicite :

42. P. 892.

43. Il faut dire aussi qu'un autre type de "merveilleux " très moderne se développe dans les Mémoires : celui de la singularité humaine et du miracle de l'individu qui suscite chez le mémorialiste de véritables extases en forme de portraits.

44. I, p. 536. 
Le roi arriva de bonne heure, se promena fort et loua beaucoup. Il fit après entrer d'Antin chez $\mathrm{M}^{\mathrm{me}}$ de Maintenon avec lui qui lui montra le plan de tout Petit-Bourg. Tout en fut approuvé, excepté une allée de marronniers qui faisait merveilles au jardin et à tout le reste, mais qui ôtait la vue de la chambre du roi. D'Antin ne dit mot, mais le lendemain matin le roi, à son réveil, ayant porté la vue à ses fenêtres, trouva la plus belle vue du monde, et non plus d'allée ni de traces que s'il n'y en eût jamais eu où elle était la veille; ni de traces de travail ni de passage dans toute cette longueur, ni nulle part auprès, que si elle n'eût jamais existé. Personne ne s'était aperçu d'aucun bruit, d'aucun embarras, les arbres étaient disparus, le terrain uni au point qu'il semblait que ce ne pouvait être que l'opération de la baguette de quelque fée bienfaisante du château enchantés ${ }^{45}$.

Des images incongrues ou pittoresques relèvent du même surgissement insolite ou humoristique de l'univers du conte dans la prose historique des Mémoires. Il est impossible de relever tous les détails, et leur identification même garde souvent une part de subjectivité. Ainsi, dans la chronique de I696, la petite taille de la duchesse de Bourgogne suscite un détail amusant qui l'assimile plaisamment à une sorte de lutin ou de personnage miniaturisé comme l'ogre transformé en souris du Chat botté : "Le roi menait la princesse qui semblait sortir de sa poche ${ }^{46}$. " À la mort de Barbezieux, en forme de cérémonie funèbre, une scène épouvantable qui semble tout droit sortie d'un conte (ou d'un cauchemar), mais ici on penserait surtout, et de manière un peu anachronique, aux monarques ubuesques d'Alice au pays des merveilles, voit Louis XIV, espèce d'ogre pour l'occasion, ordonner une sinistre scène :

[...] le Roi témoigna une joie qui parut vouloir être imitée. Il ne se contenta pas de crier : la reine boit! mais, comme en franc cabaret, il frappa et fit frapper chacun de sa cuiller et de sa fourchette sur son assiette, ce qui causa un charivari fort étrange, et qui à reprises dura tout le souper ${ }^{47}$.

Lauzun ou Monsieur le prince, dans de nombreux passages où ils sont presque interchangeables (en dehors de leur différence de rang!), s'assimilent souvent à des personnages de contes dans les anecdotes où ils endossent le rôle d'un "diablotin » ou d'une espèce de "Scapin » maléfique jouant des tours à ses victimes, comme dans une anecdote très amusante à propos de Toussaint Rose, secrétaire du cabinet de Louis XIV, extraordinaire personnage qui produit une véritable déferlante d'anecdotes comiques :

45. III, p. 35 .

46. I, p. 340 .

47. I, p. 80I. 
Il avait fort près de Chantilly une belle terre et bien bâtie qu'il aimait fort, et où il allait souvent. Il rendait force respects à Monsieur le Prince (c'est du dernier mort dont je parle), mais il était attentif à ne s'en pas laisser dominer chez lui. Monsieur le Prince, fatigué d'un voisinage qui le resserrait, et peut-être plus que lui, ses officiers de chasse, fit proposer à Rose de l'en accommoder; celui-ci n'y voulut jamais entendre, ni s'en défaire pour quoi que ce fût. À la fin Monsieur le Prince, hors de cette espérance, se mit à lui faire des niches pour le dégoûter et le résoudre, et de niche en niche, il lui fit jeter trois ou quatre cents renards ou renardeaux qu'il fit prendre et venir de tous côtés, par-dessus les murailles de son parc. On peut se représenter quel désordre y fit cette compagnie, et la surprise extrême de Rose et de ses gens d'une fourmilière inépuisable de renards venus là en une nuit. Le bonhomme, qui était colère et véhément, ne se méprit pas à l'auteur du présent $[\ldots]^{48}$.

Ce sont parfois des contrastes sociaux qui suscitent les moments où l'anecdote joue avec l'univers de référence du conte de fées. Ainsi, le fils pauvre du marquis du Châtelet, "brave et honnête garçon, mais aussi demeuré que le père ", épouse-t-il comme miraculeusement la fille d'un duc et pair, la révélation de cet extraordinaire coup de fortune occasionnant un passage très drôle sur la stupeur des parents du jeune homme et un happy end qui voit la "vertu [...] récompensée 49 ». Dans un passage encore plus amusant, le prince de Léon semble décrit, en focalisation interne, et plus précisément à travers les yeux d'une femme de chambre, comme un prince charmant, " un beau monsieur tout jeune, tout doré, frisé et poudréso ".

Dans un magnifique passage de l'année 1705, des chasseurs se perdent dans la forêt, errent de nuit, et parviennent à une maison où ils vont découvrir Fargues vivant comme un ermite ! Certains détails semblent surgir directement de l'univers du Petit Poucet:

[ils] s'égarèrent ; et les voilà à la nuit noire à ne savoir où ils étaient. À force d'aller sur leurs chevaux recrus, ils avisèrent une lumière; ils y allèrent, et à la fin arrivèrent à la porte d'une espèce de château ${ }^{5 \mathrm{I}}$. Ils frappèrent, ils crièrent, ils se nommèrent, et demandèrent l'hospitalité. C'était à la fin de l'automne, et il était entre dix et onze heures du soir. On leur ouvrit. Le maître vint au-devant d'eux, les fit débotter et chauffer, fit mettre leurs chevaux dans son écurie, et pendant ce temps-là leur fit préparer à souper $[\ldots]^{52}$.

48. I, p. 804 .

49. IV, p. 762 pour tous ces passages.

50. III, p. I60.

5I. Yves Coirault remarque en note, en II, p. I479-I480, le caractère archétypal de ces lieux : forêt obscur, château, au-delà du " réalisme " apparent de la scène.

52. II, p. 633. 
Ailleurs, l'univers du conte envahit les anecdotes saint-simoniennes de manière toujours éclatée, mais saisissante, révélant à quel degré de profondeur le " réel " peut être envisagé à travers les modèles fournis par la fiction et intégré verbalement dans les "moules " langagiers qu'elle propose, dans une parfaite osmose entre imaginaire du monde et imaginaire des discours. Dans certains cas, il devient impossible de dire si les traces d'univers du conte éparses dans l'œuvre restent conscientes et parodiques ou si elles trahissent le manichéisme régressif et l'esprit de superstition qui menacent sans cesse les Mémoires ${ }^{53}$. Les anecdotes relatives au père de l'auteur, dans la vaste digression de l'année i693 qui lui est consacrée à l'occasion de sa mort, regorgent de traits de ce genre, à la fois parce que la question du père est un puissant facteur " régressif ${ }^{54}$ " et parce que les anecdotes proviennent d'un Saint-Simon enfant auditeur de son père et ont d'une certaine manière la saveur de " contes du temps jadis ". Deux exemples : c'est de manière quasi-miraculeuse et sans autre explication qu'une anecdote de chasse grotesque que le père " devint tout à coup favori » de Louis XIII "sans autre protection que la bonté seule du Roiss", la parenté de cet événement avec un prodige de conte tenant évidemment à la naïveté caricaturale et à la crédulité de Saint-Simon sur tout ce qui touche à l'origine " fabuleuse " à ses yeux du duché-pairie. Un passage relatif au premier mariage du duc Claude est encore plus significatif, à propos de deux sœurs filles du duc de Montmorency entre lesquelles son père est censé hésiter, et qui, par leur contraste trop ridiculement tranché pour être "vrai ", font penser aux Fées ou à Cendrillon :

Il avait laissé deux filles extrêmement différentes, une Lia et une Rachel. L'aînée était également laide, méchante, glorieuse, artificieuse ; la cadette belle et agréable au possible, avec une douceur, une bonté et des agréments qui ne firent que rehausser sa vertu, et qui la firent aimer de tout le monde. Ce fut celle que mon père choisit ${ }^{56}$.

On a du mal à croire que Saint-Simon adhère à une vision aussi rudimentaire du monde "réel ", mais, face à ces " récits de l'origine ", son sens critique n'agit pas du tout et l'univers du conte s'empare avec aisance d'un passé qui n'existe pour lui que par les récits de son père qui l'y avaient sans doute déjà, pour une large part, fait basculer.

53. Sur ce point, voir les analyses d'Alphonse de Waelhens dans Le duc de Saint-Simon, Immuable comme Dieu et d'une suite enragée, Publications des Facultés universitaires Saint-louis, Bruxelles, I98I, et en particulier le chapitre "le renversement manichéen ", p. 295-309.

54. Toujours chez Waelhens, chapitre «L'origine et le père ", p. I7-28.

55. I, p. 59.

56. I, p. 76-77. 
On voit ici se dégager une zone où « l'influence» du conte merveilleux ne participe plus du tout d'une mise à distance ironique d'un genre envisagé de haut, du fait de son origine populaire, par l'homme de cour : Saint-Simon semble au contraire avoir un besoin naif de traiter cette " réalité » qui lui tient tant à cœur sur le modèle du conte, comme pour l'affubler d'une simplicité de sens coïncidant avec les visions archétypales de sa "scène intérieure ${ }^{57}$ ». On pourrait dire que, comme toute une partie de l'élite de son époque, Saint-Simon s'offre le double plaisir de se moquer du conte et d'en jouir pleinement et au "premier degré » malgré tout, de faire du conte le lieu d'un jeu distancié et ironique présentable socialement et tout à la fois d'une plongée rafraîchissante dans un univers aux oppositions si tranchées qu'elles coïncident plus que la réalité avec un monde intérieur qui ne s'encombre pas de ses nuances. Pour ces raisons, il est parfois difficile, lorsque l'univers du conte vient hanter l'anecdote, dans les Mémoires, de faire la part de la dérision et du pur plaisir de " conter». Une des plus belles anecdotes des premières années des Mémoires, à propos de Charnacé, ancien page du Roi arrêté pour fausse monnaie après avoir "fait bien des fredaines ", me semble résumer de manière particulièrement saisissante cette ambiguïté :

Il en fit une ${ }^{58}$, entre autres, pleine d'esprit et dont on ne put que rire. Il avait une très longue et parfaitement belle avenue devant sa maison en Anjou, dans laquelle était placée une maison de paysan et son petit jardin, qui s'y était apparemment trouvée lorsqu'elle fut plantée, et que jamais Charnacé ni son père n'avaient pu réduire ce paysan à la leur vendre, quelque avantage qu'ils lui en eussent offert; et c'est une opiniâtreté dont quantité de petits propriétaires se piquent pour faire enrager des gens à la convenance et quelquefois à la nécessité desquels ils sont. Charnacé, ne sachant plus qu'y faire, avait laissé cela là depuis très longtemps sans en plus parler. Enfin, fatigué de cette chaumine qui lui bouchait tout l'agrément de son avenue, il imagina un tour de passe-passe. Le paysan qui y demeurait, et à qui elle appartenait, était tailleur de son métier quand il trouvait à l'exercer, et il était chez lui tout seul sans femme ni enfants. Charnacé l'envoie chercher, lui dit qu'il est mandé à la cour pour un emploi de conséquence, qu'il est pressé de s'y rendre, mais qu'il lui faut une livrée. Ils font marché comptant ; mais Charnacé stipule qu'il ne veut point se fier à ses délais et que, moyennant quelque chose de plus, il ne veut point qu'il sorte de chez lui que sa livrée ne soit faite, et qu'il le couchera, le nourrira et le payera avant de le renvoyer. Le tailleur s'y accorde et se met à travailler. Pendant qu'il y est occupé, Charnacé fait prendre avec la dernière exactitude le plan et les dimensions de sa maison et de son jardin, des pièces de l'intérieur, jusque de la position des ustensiles et du petit meuble, fait démonter la maison et emporter tout ce qui y était, remonte

57. Voir encore sur ce point les pénétrantes analyses d'Alphonse de Waelhens.

58. Une de ses « fredaines »... 
la maison telle qu'elle était au juste, dedans et dehors, à quatre portées de mousquet à côté de son avenue, replace tous les meubles et ustensiles dans la même position en laquelle on les avait trouvés, et rétablit le petit jardin de même ; en même temps fait aplanir et nettoyer l'endroit de l'avenue où elle était, en sorte qu'il n'y parût pas. Tout cela fut exécuté encore plus tôt que la livrée faite, et cependant le tailleur doucement gardé à vue de peur de quelque indiscrétion. Enfin, la besogne achevée de part et d'autre, Charnacé amuse son homme jusqu'à la nuit bien noire, le paye et le renvoie content. Le voilà qui enfile l'avenue : bientôt, il la trouve longue ; après, il va aux arbres et n'en trouve plus. Il s'aperçoit qu'il a passé le bout, et revient à tâtons chercher les arbres. Il les suit à l'estime, puis croise et ne trouve point sa maison. Il ne comprend point cette aventure. La nuit se passe dans cet exercice ; le jour arrive et devient bientôt assez clair pour aviser sa maison : il ne voit rien ; il se frotte les yeux, il cherche d'autres objets pour découvrir si c'est la faute de sa vue. Enfin, il croit que le diable s'en mêle, et qu'il a emporté sa maison. À force d'aller, de venir, et de porter sa vue de tous côtés, il aperçoit à une assez grande distance de l'avenue une maison qui ressemble à la sienne comme deux gouttes d'eau. Il ne peut croire que cela soit; mais la curiosité le fait aller où elle est, et où il n'a jamais vu de maison. Plus il approche, plus il reconnaît que c'est la sienne. Pour s'assurer mieux de ce qui lui tourne la tête, il présente sa clef ; elle ouvre ; il entre : il retrouve tout ce qu'il y avait laissé, et précisément dans la même place. Il est prêt à en pâmer, et il demeure convaincu que c'est un tour de sorcier. La journée ne fut pas bien avancée que la risée du château et du village l'instruisit de la vérité du sortilège, et le mit en furie : il veut plaider, il veut demander justice à l'intendant; et partout on s'en moque. Le Roi le sut qui en rit aussi, et Charnacé eut son avenue libre. S'il n'avait jamais fait pis, il aurait conservé sa réputation et sa libertés9.

On devine qu'il s'agit ici une des rarissimes pages des Mémoires à donner à un paysan, ou à tout autre homme du peuple, une place de premier plan dans la narration : cette anecdote est donc extraordinaire à double titre, par le faux sortilège qui lui donne sa saveur, mais aussi par le personnage inhabituel qui est mis en vedette. L'ironie est perceptible : c'est celle d'un homme de cour s'adressant (en principe) à d'autres membres de l'élite, sur le dos d'un homme " de la lie du peuple » impitoyablement tourné en dérision. En ce sens, le « conte de fées " que semble vivre de l'intérieur le paysan est le revers déconsidéré de l'anecdote brillante d'un " courtisan ", il faut le dire, particulièrement inspiré, le récit possible qu'on aurait pu faire si on avait adhéré à l'univers de croyance du paysan, à sa " naïveté ». Mais les choses ne sont pas si simples et l'ironie n'explique pas tout : il est d'abord fascinant que, dans une logique qu'on pourrait généraliser à propos des Mémoires, le style du mémorialiste semble "dialoguer " avec une langue de "paysan " réelle ou " reconstituée ", comme si Saint-Simon ne pouvait parler de ce personnage qu'en lui faisant, au moins fantasma-

59. I, p. 524-526. 
tiquement, co-narrer son éblouissant récit. Il y a ainsi, dans la partie la plus remarquable de l'anecdote, par la simplicité du style et de la syntaxe, par la " naïveté " des tours, des passages qu'on aurait envie de "transcrire " à la première personne : " après, je vais aux arbres et n'en trouve plus. Je m’aperçois que j'ai passé le bout [...]", " je ne vois rien ; je me frotte les yeux », etc., comme pour les restituer à une origine " populaire » au niveau de l'énonciation. En outre, le contexte nocturne, le cadre "forestier ", l'errance du personnage dans un tel univers, et d'un personnage qui n'apparaît guère ailleurs que dans les contes dans la galaxie des récits d'Ancien Régime que pouvait connaître Saint-Simon, apparaissent comme autant de signes supplémentaires d'un "dialogue " pour une part ironique, pour une part porté par le pur plaisir de conter, entre les festivités stylistiques d'une somptueuse anecdote de cour et la "naïveté populaire " du conte archétypal. Ce n'est évidemment pas un hasard si une des rares scènes des Mémoires à faire d'un paysan un personnage important se colore, dans l'imaginaire linguistique et thématique qu'elle véhicule, de bien des marques d'un genre populaire dans son origine réelle ou fantasmée. On peut donc soupçonner Saint-Simon d'écrire, dans la mesure où on tient à ces catégories, à la fois une anecdote et un conte, la première étant chargée de recouvrir d'un voile d'ironie mondaine et critique la naïveté, la « fraîcheur » irrésistible (et secrètement savourée) du second.

Le conte me semble donc entretenir avec l'anecdote, dans les Mémoires de Saint-Simon, œuvre d'histoire, œuvre de savoir, œuvre d'érudition même, et œuvre de vérité, un rapport (paradoxalement) privilégié pour deux raisons principales : la première tient à l'extraordinaire succès concomitant de ces deux "genres " à l'époque classique, et à l'imitation par l'élite courtisane des jeux savants de la " littérature " avec des formes narratives perçues au départ comme " populaires ». Saint-Simon reprend ces jeux à son compte et fait " résonner " de manière étourdissante la parole d'un conteur archétypal à l'arrière-plan de l'énonciation "versaillaise ". La seconde tient à la nature même de ces deux modèles fondamentaux de la narration brève, qui semblent faits pour fusionner et pour s'emprunter mutuellement des ressources, puisque le « conte» veut (parfois) avoir l'air vrai, et que l'anecdote veut plaire, ce qui les rapproche " asymptotiquement " sur le plan de leurs formes ${ }^{60}$. Mais les questions formelles ou

6o. La question du «format » est ici loin d'être secondaire, car le conte comme l'anecdote doit pouvoir s'intégrer dans un échange et participer du "flux de bouche " (j'emprunte l'expression à Saint-Simon...) d'une " conversation " que les Mémoires semblent vouloir prolonger, au moins imaginairement, dans l'étrange face à face de leur auteur avec l'écriture et avec la mort. 
"poétiques» rencontrent ici directement les questions anthropologiques, et notamment des questions de croyance. Plus profondément, représenter le monde, pour Saint-Simon, c'est faire entendre toutes ses voix, la frontière entre " représentation " et " énonciation " n'étant pas ici opératoire, et la voix des " conteurs " et des contes fait partie, dans la polyphonie de son œuvre, de celles qu'il ne pouvait pas ne pas faire entendre. L'intuition du narrateur proustien, qui associe les Mémoires de Saint-Simon et les Mille et Une Nuits dans cette espèce d'archéologie imaginaire de son œuvre à venir du Temps retrouvé, me semble donc marquer ce lieu magique et rare où la frontière pourtant si essentielle, si vitale, entre fiction et histoire, momentanément abolie, s'évanouit dans la pure féerie du récit. 